\title{
Uso de páginas Web u otros medios para la prevención de enfermedades crónicas no transmisibles
}

\section{Use of websites or other sources of information for the prevention of chronic non-communicable diseases}

\author{
Antonio Alan Alves 1,a ${ }^{1}$, Danitza Adriana Acuña Rodríguez 1,b
}

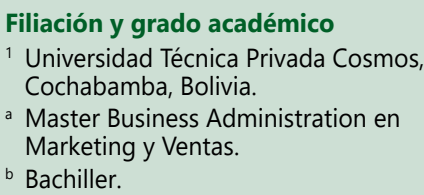

AA: concepción y diseño del trabajo, análisis e interpretación de los datos, redacción del manus- crito, revisión crítica del manuscrito, aprobación de la versión final.

DAAR: concepción y diseño del trabajo/ idea, re- dacción del manuscrito, revisión crítica del ma- nuscrito, aprobación de la versión final.

Fuentes de financiamiento La investigación fue realizada con recursos propios.

Conflictos de interés

Los autores declaran no tener conflictos de interés.

Recibido: 30-10-2020

Arbitrado por pares

Aceptado: 31-12-2020

Citar como

Alan Alves A, Acuña Rodríguez DA. Uso de páginas Web u otros medios para la prevención de enfermedades crónicas no transmisibles. Rev Peru Cienc Salud. 2021; 3(1): 8-12 doi: https://doi.org/10.37711/ rpcs.2021.3.1.242

Correspondencia

Antonio Alan Alves

Dirección: Avenida Ramon Rivero,

Cochabamba, Bolivia. CP. 2500

Telf.: +59179776510

Email: alan29.6@gmail.com

\section{RESUMEN}

Objetivo. Describir la frecuencia de uso de páginas Web o medios digitales para la prevención de enfermedades crónicas no transmisibles en la ciudad de Cochabamba, Bolivia. Métodos. Participaron en el estudio 303 personas clasificadas de acuerdo con el sexo: 156 mujeres y 147 hombres; en relación con la vivienda: 279 habitantes de Cochabamba y 24 de otros lugares y de acuerdo con el grado de colegiatura: 208 con nivel universitario, 90 con nivel secundario y 5 con otros grados de colegiatura. Fueron evaluadas 63 páginas Web que atendían a los requisitos de tener, por lo menos, un puntaje de 60 puntos en la hoja de cotejo. Resultados. 227 personas recurrían al medio digital para obtener informaciones sobre ENT, 27 recurrían a los puestos de salud (públicos), 32 a las clínicas particulares y las 17 personas restantes recurrían a médicos tradicionales, amigos, parientes $u$ otros medios. De las 63 páginas Web evaluadas, todas tenían facilidad de acceso, 31,7 \% tenían un buen diseño y una buena interacción con el usuario y solo $62,4 \%$ tenían contenidos satisfactorios. Conclusiones. Los resultados evidencian que la mayoría de las personas encuestadas $(74,9 \%)$ recurren al medio digital como fuente de información sobre prevención de ENT, pero, infelizmente, las páginas Web no brindan información completa, segura y tampoco confiable para los usuarios.

Palabras clave: mercadeo; internet; prevención primaria; enfermedades no transmisibles; promoción de la salud (Fuente: DeCS-BIREME).

\section{ABSTRACT}

Objective. Describe the frequency of use of websites or digital media for the prevention of chronic non-communicable diseases in the city of Cochabamba, Bolivia. Methods. The study involved 303 persons classified according to sex: 156 women and 147 men; in relation to housing: 279 inhabitants of Cochabamba and 24 from other places and according to the degree of education: 208 with university level, 90 with high school level and 5 with other degrees of education. 63 websites were evaluated that met the requirements of having at least a score of 60 points on the checklist. Results. 227 people used the digital media to obtain information on NCDs, 27 used (public) health posts, 32 used private clinics and the remaining 17 people used traditional doctors, friends, relatives or other means. Of the 63 websites evaluated, all have easy access, $31.7 \%$ have a good design and interaction with the user and only $62.4 \%$ have satisfactory content. Conclusions. The results show that the majority of the people surveyed (74.9\%) use the digital medium as a source of information on NCD prevention but, unfortunately, the websites do not provide complete, safe and reliable information for users.

Keywords: marketing; internet; primary prevention; non-communicable diseases; health promotion (Source: MeSH - NLM). 


\section{INTRODUCCIÓN}

Antiguamente se pensaba en medios digitales desde una perspectiva demasiado cerrada pues, en ese entonces, era más que suficiente lograr unos cuantos likes; lo que ya no es aceptable en la actualidad. La evolución ha demostrado que el potencial de estos medios es mucho más amplio, sobre todo en la perspectiva del poder que tiene en transformar hábitos de vida, logrando una estrategia base para la atención primaria de salud ${ }^{(1,2)}$.

En 1997, la declaración de Yakarta sobre promoción de la salud en el siglo XXI indicó que la capacidad de las comunidades para la promoción de la salud requería nuevas tecnologías de información y comunicación (TIC) que, en un principio, estaban limitadas a la radio, televisión y telefonía, mientras que actualmente están conformadas por todas estas, sumadas a los smartphones y computadoras que tienen como vehículo de comunicación las páginas Web, ya que estas tienen un papel fundamental para fomentar la promoción de la salud y la prevención de las enfermedades ${ }^{(3-5)}$.

Son muchos los beneficios proporcionados por las páginas Web, pero también hay perjuicios. En un mundo de saturación de información, donde las personas no están seguras de qué información es fiable, las instituciones tienen como obligación promocionar la salud e informar sobre la prevención de las enfermedades de manera efectiva ${ }^{(6-7)}$.

En el caso de las enfermedades crónicas no transmisibles (ENT) que tienden a ser de largo plazo y son el resultado de la combinación de factores fisiológicos, genéticos, ambientales y de estilo de vida que causan más de 41 millones de decesos por año, lo que equivale al $71 \%$ de las muertes que se producen en el mundo, estas son, sin embargo, un grupo de enfermedades que se pueden prevenir ${ }^{(8-11)}$.

Las ENT que afectan al mayor número de personas son las enfermedades cardiovasculares, accidentes cerebrovasculares, cáncer, diabetes, enfermedad pulmonar obstructiva crónica (EPOC) y asma. Estas enfermedades suelen asociarse a los grupos etarios más avanzados, pero los datos muestran que 15 millones de todas las muertes provenientes de las ENT se producen entre los 30 y 69 años ${ }^{(10,12,13)}$.

El objetivo de este trabajo es describir la frecuencia de uso de páginas Web o medios digitales para la prevención de enfermedades crónicas no transmisibles en la ciudad de Cochabamba, Bolivia.

\section{MÉTODOS}

\section{Tipo de estudio}

Enfoque cualitativo, descriptivo y de corte transversal, con población infinita, basado en los habitantes del municipio de Cochabamba, Bolivia. El estudio se desarrolló en el primer semestre de 2019.

\section{Población y muestra}

En este estudio participaron 303 personas residentes de Cochabamba con un rango etario de 14 hasta 62 años. Los criterios de exclusión fueron ser personas menores de 14 años, mayores de 62 años y personas que no residieran en Cochabamba. Para determinar la muestra se utilizó la formula estadística de población infinita $\mathrm{N}=(\mathrm{Za})^{2} \times(\mathrm{p} \times \mathrm{q}) / \mathrm{d}^{2}, \mathrm{Za}=95 \%, \mathrm{p}=50 \%, \mathrm{q}=$ $50 \%$ y $d=6 \%$. Para determinar el ajuste de muestra se utilizó la formula estadística: $n=N \times 1 /(1-R)$, en donde la muestra ajustada fue de 303 personas; se utilizó un muestreo aleatorio simple.

\section{Instrumentos de recolección de datos}

Las personas participantes junto a la unidad de información que fueron las páginas Web, de las cuales se obtuvieron, por lo menos, 60 de los 100 puntos en la hoja de cotejo; luego, fueron sometidas a evaluación según los criterios de Caywood ${ }^{(14)}$. Cuestionarios digitales: encuesta (con preguntas de identificación y preguntas específicas) y hoja de cotejo (con tres criterios para selección de páginas Web.

\section{Procedimientos de la recolección de datos}

La recolección de datos fue desarrollada por medio de cuestionarios digitales, en donde los usuarios recibieron la encuesta por la aplicación de WhatsApp ${ }^{\oplus}$ y, asimismo, fue compartida con colegas y familiares. La encuesta presentó preguntas de identificación personal: nombre, sexo, edad, municipio de residencia y grado de colegiatura. Preguntas específicas sobre el grado de conocimiento sobre ENT y de selección múltiple sobre el medio utilizado para la obtención de información sobre prevención de ENT. La hoja de cotejo presentó tres criterios para la selección de las páginas Web, que fueron: la página se encuentra entre las 3 primeras páginas del buscador Google ${ }^{\circledast}$; de acuerdo a las palabras claves seleccionadas, la página se encuentra entre las 3 primeras páginas del buscador Yahoo Search ${ }^{\oplus}$ de acuerdo a las palabras claves seleccionadas y la página se encuentra entre las 3 primeras páginas del buscador Bing Search ${ }^{\oplus}$; de acuerdo con las palabras claves seleccionadas y luego las páginas Web seleccionadas fueron evaluadas según los criterios de Caywood (ver tabla 1). Las palabras claves utilizadas fueron: prevención de enfermedades cardiovasculares, 
Sexo

303 respostas

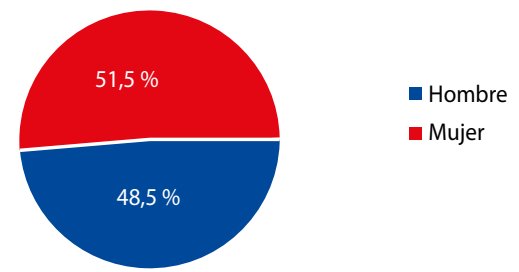

Figura 1. Clasificación de acuerdo al sexo

prevención de accidentes cerebrovasculares, prevención de cáncer, prevención de diabetes, prevención de EPOC y prevención de asma.

\section{Aspectos éticos}

Este trabajo se adecua a las normas establecidas en el capítulo XIX del Código de Ética y Deontología Médica del Ministerio de Salud de Bolivia ${ }^{(15)}$. El consentimiento informado se realizó de forma escrita para todos los participantes.

\section{RESULTADOS}

Participaron 303 personas clasificadas de acuerdo al sexo: 156 mujeres $(51,5 \%$ ) y 147 hombres (48,5\%) (ver figura 1) y de acuerdo con el grado de colegiatura: 208 $(68,6 \%)$ con nivel universitario, $90(29,7 \%)$ con nivel secundario y $5(1,7 \%)$ con otros grados de colegiatura (ver figura 2).

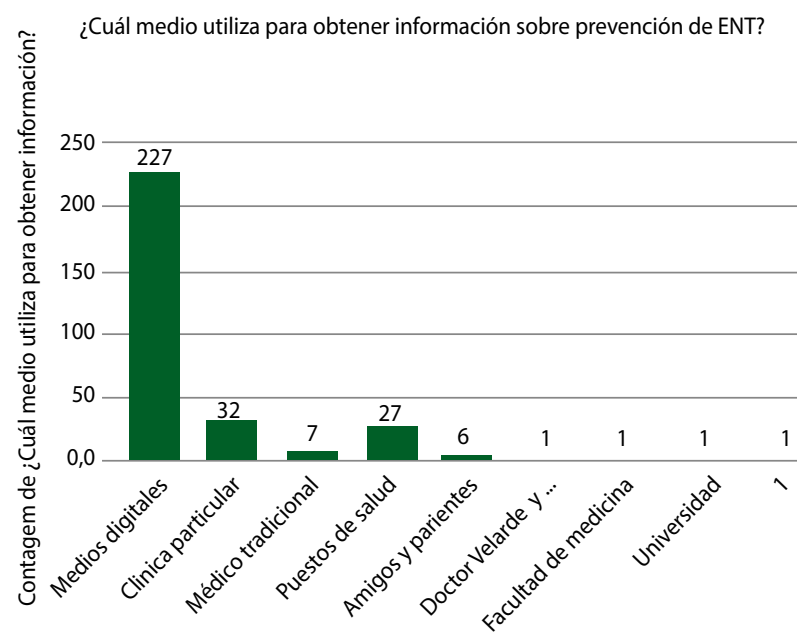

Figura 3. Medios para obtención de información sobre prevención de ENT
Grado de colegiatura

303 respostas

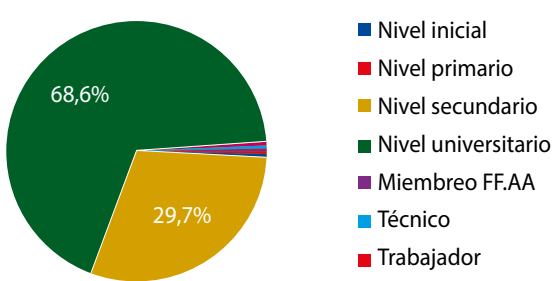

Figura 2. Clasificación de acuerdo al grado de colegiatura

Cuando se preguntó cuál medio utiliza el participante para obtener información sobre prevención de ENT, 227 $(74,9 \%)$ personas expresaron que recurren al medio digital, 32 (10,6\%) recurren a las clínicas particulares, 27 $(8,9 \%)$ recurren a los puestos de salud (públicos) y los $17(5,6 \%)$ restantes recurren a médicos tradicionales, amigos, parientes y otros medios (ver figura 3).

\section{DISCUSIÓN}

En pleno siglo XXI, las TIC son imprescindibles para llevar contenido a las poblaciones más alejadas, además de ser una herramienta fundamental tanto para los pacientes como para los profesionales del área de la salud e instituciones afines ${ }^{(16,17)}$.

Obviamente aún falta entrenamiento de estos profesionales 0 , simplemente, un equipo multidisciplinario en centros de salud para difundir información de calidad

Tabla 1. Evaluación de 63 páginas Web según criterios de Caywood

\begin{tabular}{|c|c|c|}
\hline Criterios de caywood & Paginas & $\%$ \\
\hline \multicolumn{3}{|l|}{ Facilidad de acceso } \\
\hline \multicolumn{3}{|l|}{ Resultados en Google ${ }^{\circledast}$ (63 páginas) } \\
\hline Resultados en Yahoo Search ${ }^{\circledast}$ (63 páginas) & 63 & 100 \\
\hline \multicolumn{3}{|l|}{ Resultados en Bing Search ${ }^{\circledast}$ (63 páginas) } \\
\hline \multicolumn{3}{|c|}{ Diseño e interacción con el usuario (promedio) } \\
\hline Presenta imágenes (38 páginas) & 20 & 31,7 \\
\hline \multicolumn{3}{|l|}{ Presenta videos (2 páginas) } \\
\hline \multicolumn{3}{|l|}{ Calidad del contenido (promedio) } \\
\hline \multicolumn{3}{|l|}{ Presenta introducción (51 páginas) } \\
\hline Presenta prevención (52 páginas) & 39,3 & 62,4 \\
\hline \multicolumn{3}{|l|}{ Presenta bibliografía (15 páginas) } \\
\hline Evaluación final promediada & $64,7 \%$ & \\
\hline
\end{tabular}


por las páginas Web; así también, implementar una traducción para su idioma nativo, ya que, en Cochabamba el $47 \%$ de la población es quechuahablante ${ }^{(18-20)}$.

Los resultados de este estudio demuestran, por consiguiente, que la población de Cochabamba prefiere buscar información sobre prevención de ENT en el medio digital; por lo cual, las instituciones tienen una excelente herramienta que debe ser explorada, llevando información de calidad a todos los hogares de las familias cochabambinas.

Un estudio realizado por universidades españolas también evidencia que los medios digitales son una excelente oportunidad para informar y promover campañas de salud con un gran público; lo que demuestra que esta es una tendencia mundial ${ }^{(21)}$.

Mientras no se dé prioridad a mejorar la calidad de la información, se recomienda a la población en general a buscar información en más de una página Web y compararla para así obtener una información completa y fiable ${ }^{(22,23)}$.

\section{REFERENCIAS}

1. American Marketing Association. Definition of marketing [Internet] American Marketing Association; 2013 [Consultado 2019 Mar 25] Disponible en: https://www. ama.org/the-definition-of-marketing/

2. Rodríguez SC, Cabrera LC, Calero Yera E. La comunicación social en salud para la prevención de enfermedades en la comunidad. Rev Hum Med [Internet] 2018 Ago; 18(2): 384-404 [Consultado 2019 Mar 25] Disponible en: http://scielo.sld.cu/scielo.php?script=sci_arttext\&pi$\mathrm{d}=\mathrm{S} 1727-81202018000200384 \&$ Ing=es\&nrm=iso

3. García H, Navarro L, López M, Rodríguez MF. Tecnologías de la Información y la Comunicación en salud y educación médica. EDUMECENTRO [Internet] 2014; 6(1) [Consultado 2019 Mar 25]] Disponible en: http://scielo.sld.cu/scielo. php?script=sci_arttext\&pid=S2077-28742014000100018

4. La importancia de las TIC en el sector educación [Internet] Universia México; 2020 [Consultado 2021 Ene 7] Disponible en: https://www.universia.net/mx/actualidad/orientacion-academica/importancia-tic-sector-educacion-1129074.html

5. Organización Mundial de la Salud. Declaración de Yakarta sobre la Promoción de la Salud en el Siglo XXI [Internet] [Consultado 2021 Ene 7] Disponible en: https:// www.who.int/healthpromotion/conferences/previous/ jakarta/en/hpr_jakarta_declaration_sp.pdf

6. Caja Costarricense de Seguro Social. Curso Especial de Posgrado Atención Integral de Salud [Internet] [Consultado 2021 Ene 7] Disponible en: https://montevideo.gub.uy/sites/default/files/concurso/documentos/ Ed211.paralaSalud-MariaT.Cerqueira.pdf

7. Estudio de Comunicación, S.A. Comunicación. Soluciones para un mundo [Internet] [Consultado 2021 Ene 7]
Disponible en: https://www.estudiodecomunicacion. com/wp-content/uploads/2018/01/Comunicacion.-Soluciones-para-un-mundo-digital.pdf

8. Domínguez $E$, Seuc A. Esperanza de vida ajustada por algunas enfermedades crónicas no transmisibles. Rev Cubana Hig Epidemiol. [Internet] 2005 Ago; 43(2) [Consultado 2021 Ene 7] Disponible en: http://scielo.sld.cu/scielo.php?script=sci_arttext\&pi$d=S 1561-30032005000200006$

9. Cahuana-Berrocal J, Donado-Gamez G, Barroso-Martínez L, González-Redondo N, Lizarazu-Diazgranados I, Iglesias-Acosta J. Epigenética y Enfermedades Crónicas no Transmisibles. iMedPub Journals [Internet] 2019 Sep; 15(4) [Consultado 2021 Ene 7] doi: 10.3823/1419

10. Organización Mundial de la Salud. Enfermedades no transmisibles [Internet] [Consultado 2021 Ene 7] Disponible en: https://www.who.int/es/news-room/factsheets/detail/noncommunicable-diseases

11. Jadue L, Vega J, Escobar MC, Delgado I, Garrido C, Lastra $\mathrm{P}$, et al. Factores de riesgo para las enfermedades no transmisibles: Metodología y resultados globales de la encuesta de base del programa CARMEN (Conjunto de Acciones para la Reducción Multifactorial de las Enfermedades no Transmisibles). Rev. méd. Chile. [Internet] 1999 Ago; 127(8): 1004-1013. [Consultado 2021 Ene 7] Disponible en: http://dx.doi.org/10.4067/S003498871999000800017

12. González AM, Palma ME. Principales causas de morbilidad en una población de adultos mayores. Rev haban cienc méd. [Internet] 2008 Jun; 7(2) [Consultado 2021 Ene 7] Disponible en: http://www.revhabanera.sld.cu/ index.php/rhab/article/view/1411

13. Instituto Nacional de Estadística. Población en Bolivia 2018. [Internet] [Consultado 2019 Mar 19] Disponible en: https://www.ine.gob.bo/index.php/convocatorias-de-bienes-y-servicios/item/3170-bolivia-cuenta-con-mas-de-11millones-de-habitantes-a-2018

14. Ramos E. Criterios más utilizados para la evaluación de la calidad de los recursos de información en salud disponibles en Internet. ACIMED. [Internet] 2004 Apr; 12(2) [Consultado 2021 Ene 7] Disponible en: http://scielo.sld.cu/scielo.php?script=sci_arttext\&pi$\mathrm{d}=\mathrm{S} 1024-94352004000200004$

15. Ministerio de Salud de Bolivia. Código de ética y deontología médica [Internet] [Consultado 2021 Ene 13] Disponible en: https://www.minsalud.gob.bo/images/ Documentacion/normativa/CODIGODEETICAYDEONTOLOGIAMEDICA.pdf

16. Lorca J, Martí R, Albacar N, García M, Mora G, Lleixà M. Apoyo tecnológico a los cuidadores no profesionales de personas con enfermedades crónicas. Metas Enferm. [Internet] 2016 Jun; 19(5): 21-26 [Consultado 2021 Ene 7] Disponible en: https://www.enfermeria21.com/revistas/metas/articulo/80921/apoyo-tecnologico-a-los-cuidadores-no-profesionales-de-personas-con-enfermedades-cronicas/

17. Brossard F. Hacia un modelo de inclusión digital rural Una mirada sobre América Latina y el caso de Chile. Nueva Sociedad [Internet] 2016 Abr; 262(1): 97-107 [Consultado 2021 Ene 7] Disponible en: https://biblat.unam. $\mathrm{mx} / \mathrm{es} / \mathrm{revista/nueva-sociedad/articulo/hacia-un-mo-}$ delo-de-inclusion-digital-rural-una-mirada-sobre-america-latina-y-el-caso-de-chile 
18. Fernández LM, Gordo MÁ, Laso S. Enfermería y Salud 2.0: recursos TICs en el ámbito sanitario. Index Enferm. [Internet] 2016 Jun; 25(1) [Consultado 2021 Ene 7] Disponible en: http://scielo.isciii.es/scielo.php?script=sci_arttext\&pid=S1132-12962016000100012

19. Campos de Aldana MS, Moya Plata D, Mendoza Matajira JD, Duran Niño EY. Las Enfermedades Crónicas No Transmisibles Y El Uso De Tecnologías De Información Y Comunicación: Revision Sistematica. Rev Cuid. [Internet] 2014 Jun; 5(1) 661-669 [Consultado 2021 Ene 7] Disponible en: http://www.scielo.org.co/scielo.php?pid=S221609732014000100010\&script=sci_abstract\&tlng=es

20. Instituto Nacional de Estadística. Censo de Población y Vivienda 2012 [Internet] [Consultado 2021 Ene 16] Disponible en: https://bolivia.unfpa.org/sites/default/files/ pub-pdf/Caracteristicas_de_Poblacion_2012.pdf

21. Beerli-Palacio A, Martín-Santana JD, Porta M. El marketing como herramienta para incrementar la eficacia de los planes de salud pública. Gaceta Sanitaria [Internet] 2018 Abr; 22(1): 27-36 [Consultado 2021 Ene 7] Disponible en: https://www.gacetasanitaria.org/ en-el-marketing-como-herramienta-incrementar-articulo-S0213911108760722

22. Ministerio de Salud de Costa Rica. Mercadeo Social en Salud: conceptos, principios y herramientas [Internet] [Consultado 2021 Ene 13] Disponible en: http://www. fao.org/fileadmin/user_upload/red-icean/docs/Mercadeo\%20Social\%20en\%20Salud\%20PDF.pdf

23. Organización de las Naciones Unidas para la Educación, la Ciencia y la Cultura, UNESCO. Comunicación Para El Desarrollo: Fortaleciendo la eficacia de las Naciones Unidas [Internet] [Consultado 2021 Ene 13] Disponible en: http://www.unesco.org/new/fileadmin/MULTIMEDIA/ $\mathrm{HQ} / \mathrm{Cl} / \mathrm{Cl} /$ pdf/communication_form_development_ oslo_c4d_pda_es.pdf 